\title{
Long-lasting dashed waves in a reactive microemulsion
}

\author{
Jorge Carballido-Landeira, ${ }^{a}$ Igal Berenstein, ${ }^{a}$ Pablo Taboada, ${ }^{b}$ Victor Mosquera, ${ }^{b}$ \\ Vladimir K. Vanag, ${ }^{c}$ Irving R. Epstein, ${ }^{c}{ }^{\text {Vicente Pérez-Villar }{ }^{a} \text { and }}$ \\ Alberto P. Muñuzuri ${ }^{a}$
}

Received 24th September 2007, Accepted 18th December 2007

First published as an Advance Article on the web 10th January 2008

DOI: $10.1039 / b 714705 h$

\begin{abstract}
In the Belousov-Zhabotinsky (BZ) reaction carried out in a reverse microemulsion with Aerosol OT as surfactant, the existence of two different sizes of droplets containing the $\mathrm{BZ}$ reactants leads to the emergence of segmented (dashed) waves. This bimodal distribution of sizes is stabilized by adding small amounts of the homopolymer poly(ethylene oxide) (PEO). Addition of PEO lengthens the period during which these patterns are observed, so that dashed waves can persist for $12-14 \mathrm{~h}$, in contrast to the 2-3 $\mathrm{h}$ found in earlier studies without added polymer.
\end{abstract}

Pattern formation in reaction-diffusion systems has been studied in a variety of configurations including homogeneous aqueous solution, gels, membranes and resin beads. Recently, Vanag and Epstein dispersed the reactants of the BelousovZhabotinsky (BZ) reaction in an Aerosol OT (AOT) water-inoctane microemulsion, ${ }^{1}$ producing nanoscale water droplets that can communicate both through the oil phase (via oil-soluble intermediates of the $\mathrm{BZ}$ reaction, like $\mathrm{Br}_{2}$ ) and by collisions between reverse micelles. A remarkable array of new patterns has been observed in this system. One of the most striking of these consists of segmented or dashed waves, ${ }^{2,3}$ where traveling waves are broken into segments that move coherently without curling into the small spirals that usually arise in the aqueous BZ system. Dashed waves are believed to involve interaction between Hopf and Turing instabilities., ${ }^{2,3}$ These dashed waves appear only when the microemulsion is freshly prepared, at which time two distinct water droplet sizes are present. ${ }^{2}$

Acoustic spectroscopy in the water-AOT-heptane system ${ }^{4}$ reveals that two different sizes of water-containing objects appear for $\omega=\left[\mathrm{H}_{2} \mathrm{O}\right] /[$ AOT] between 50 and 80 as the system undergoes a transition from the microemulsion to an unstable turbid macroemulsion. Our own experiments with octane as the oil suggest that adding the polar contents of the BZ reaction decreases the stability of the microemulsion to phase separation and narrows the range over which the bimodal droplet distribution is found. On the other hand, poly(ethylene oxide) (PEO) stabilizes the formation of clusters in waterAOT-octane reverse microemulsions. ${ }^{5,6}$ As previously shown, ${ }^{2}$ a bimodal population distribution is found when

\footnotetext{
${ }^{a}$ Group of Nonlinear Physics, Universidade de Santiago de Compostela, Santiago de Compostela, E-15782, Spain

${ }^{b}$ Group of Colloids and Polymer Physics, Universidade de Santiago de Compostela, Santiago de Compostela, E-15782, Spain

${ }^{c}$ Department of Chemistry and Volen Center for Complex Systems, MS 015, Brandeis University, Waltham, Massachusetts 02454-9110, $U S A$
}

preparing the microemulsion system to incorporate the reactants of the BZ reaction. Without polymer, this bimodal distribution tends to a unimodal distribution with time (generally a few hours). The bimodal distribution of the "as prepared" microemulsion corresponds to single droplets of stable size and larger clusters arising from attractive interactions between droplets, the latter tending to disappear as these are non-equilibrium structures. When polymer is added, most of the polymer molecules are adsorbed on the oil/water interface of single droplets, increasing the rigidity of the interface. ${ }^{7,8}$ Some polymer molecules, however, also stabilize the droplet clusters by being shared between several droplets (the cluster), a phenomenon which occurs in other related systems. ${ }^{9}$ We note that the population of droplet clusters is significantly lower than that of single droplets. Further experiments are being developed to test this picture.

Our experiments were carried out using the same concentrations and procedure as in ref. 3: two microemulsions were prepared, one containing malonic acid (MA) and sulfuric acid and the other containing $\mathrm{NaBrO}_{3}$ and bathoferroin. The only difference was that in some experiments poly(ethylene oxide) (with molecular weight 300 or $3000 \mathrm{~g} \mathrm{~mol}^{-1}$ at a concentration of $1 \mathrm{mg}$ per $3 \mathrm{ml}$ of water phase unless otherwise indicated) was added to the microemulsion containing malonic acid and sulfuric acid. The concentrations of the $\mathrm{BZ}$ reactants in the water phase were: $[\mathrm{MA}]=0.3 \mathrm{M},\left[\mathrm{H}_{2} \mathrm{SO}_{4}\right]=0.2 \mathrm{M},\left[\mathrm{NaBrO}_{3}\right]$ $=0.18 \mathrm{M}$ and [bathoferroin] $=0.0049 \mathrm{M}$. For the reactive microemulsion, $\omega=15$ and $\varphi_{\mathrm{d}}=0.36$, where $\varphi_{\mathrm{d}}$ is the volume fraction of water droplets (including the surfactant shell).

The basic results are shown in Fig. 1. Dashed waves are obtained in both systems. In the presence of PEO, the dashed waves persist for up to $12-14 \mathrm{~h}$, compared to $3 \mathrm{~h}$ in the best of cases in the absence of the polymer. Moreover, if the microemulsions are prepared $24 \mathrm{~h}$ before starting the reaction, no dashed waves can be observed without $\mathrm{PEO},{ }^{2}$ while if PEO is added, dashed waves are present. The characteristic parameters of the patterns (wavelength, period, velocities and sizes) are the same in both cases.

In order to follow the behavior of the microemulsions, dynamic laser light scattering experiments were performed. The results are shown in Fig. 2. While for the microemulsion without PEO the two different droplet populations merge into one in $2-3 \mathrm{~h}$ (the time in which dashed waves can be observed), in the microemulsions with PEO they remain for over $12 \mathrm{~h}$.

Experiments with varying PEO 300 concentrations show that the polymer concentration influences the size of droplet 

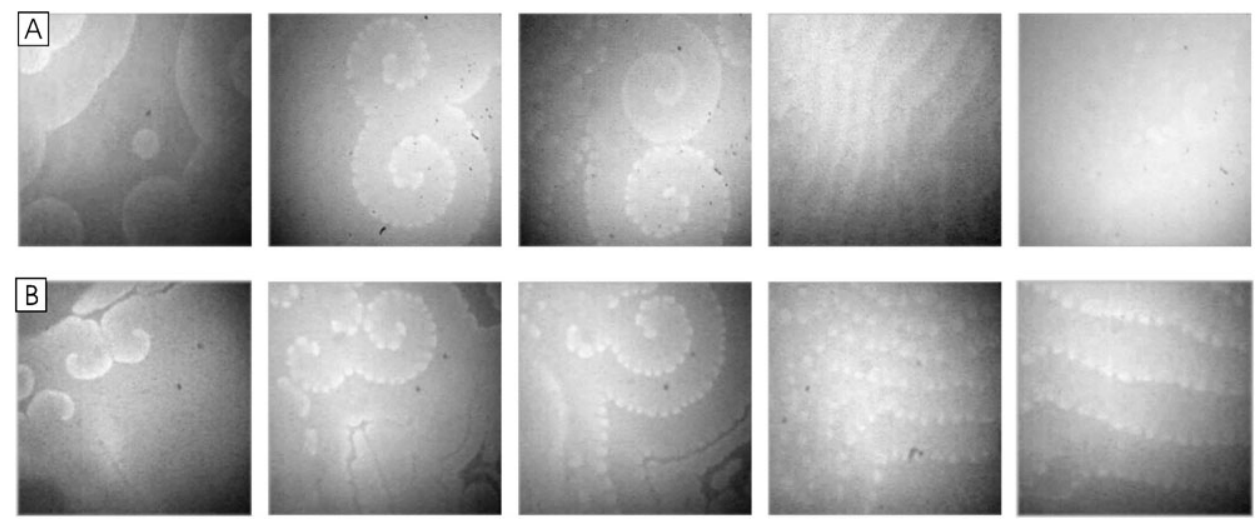

Fig. 1 Patterns obtained in the BZ-AOT system (a) without and (b) with PEO in the microemulsions. Pictures taken 40, 60, 120, 180 and 480 min (left to right) after starting the reaction. Size: $2 \mathrm{~mm} \times 2 \mathrm{~mm}$.

clusters, which decreases with PEO concentration. In addition, these clusters are smaller than those found with PEO 3000, which seems to indicate that these shorter polymer chains are shared by fewer droplets. Our experiments imply that dashed waves require the presence of at least two droplet populations with different sizes. By adding PEO to the BZ-AOT system we

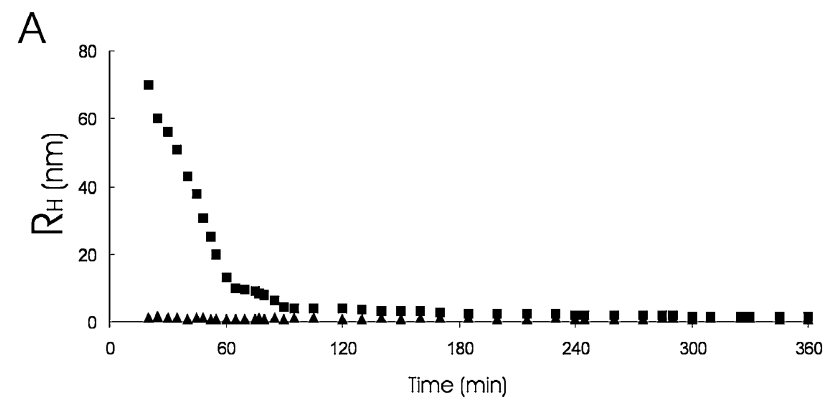

B
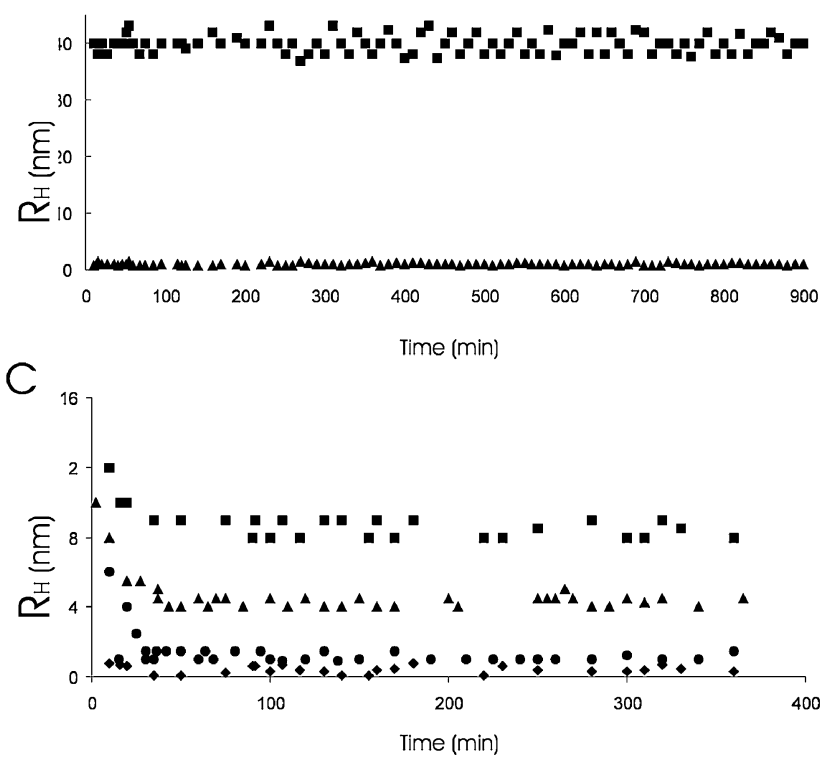

Fig. 2 Plots of hydrodynamic radius, $R_{\mathrm{H}}$, obtained from dynamic light scattering experiments of microemulsions (a) without and (b) with PEO 3000 (1 mg per $3 \mathrm{ml}$ of water phase) and (c) PEO 300 (0.23 (squares), 0.09 (triangles) and 0.0001 (circles) $\mathrm{mg}$ per $3 \mathrm{ml}$ of water phase). Microemulsions contain all $\mathrm{BZ}$ reactants except $\mathrm{FeSO}_{4}$. stabilize these populations, thereby prolonging the lifetime of the dashed waves.

On the other hand long-lasting dashed waves were observed with 0.23 and $0.09 \mathrm{mg}$ PEO 300 per $3 \mathrm{ml}$ of water phase, while long-lasting $(\sim 8 \mathrm{~h})$ normal waves (without transformation to dashed waves) were found with $0.0001 \mathrm{mg}$ of PEO 300 per $3 \mathrm{ml}$ of water phase. These results suggest that the lowest PEO 300 concentration does not give rise to distinct droplet populations with sizes sufficiently different to allow the appearance of dashed waves.

The duration of the oscillatory phase of the reaction appears to increase with the presence of PEO, as shown in Fig. 3. These experiments are carried out in a narrow cell without stirring (the optical path is $1 \mathrm{~cm}$ ), working at high concentration and hence high absorbance in order to obtain sufficient contrast for the pattern formation experiments. Lombardo and coworkers ${ }^{10}$ studied the effect of PEG (which has the same backbone structure as $\mathrm{PEO}$ ) on the $\mathrm{BZ}$ reaction, and found that the time during which oscillations could be observed was shortened by addition of the polymer, while the frequency of oscillations was increased. The same trend is found in reaction-diffusion systems. ${ }^{11}$ Thus, as previously suggested, ${ }^{12}$ confinement by the microemulsion resulting in modification of the reaction rates appears to be the origin of the increased oscillatory periods observed here.

The characteristic size $(0.1-0.5 \mathrm{~mm})$ of patterns found in the BZ-AOT system is several orders of magnitude larger than the diameter of droplets $(4-10 \mathrm{~nm})$ or clusters of droplets (40-200 $\mathrm{nm})$. Therefore, although the microemulsion structure may be

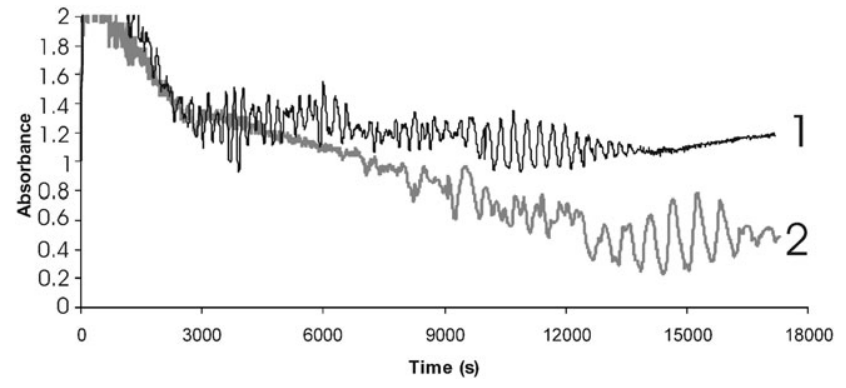

Fig. 3 Absorbance at $380 \mathrm{~nm}$ for BZ-AOT systems without (1) and with (2) PEO (1 $\mathrm{mg}$ per $3 \mathrm{ml}$ of water phase). 
affected by the BZ reactants or, as in this work, by other additives, the reaction-diffusion mechanism must be involved. In most cases, the presence of two significantly different diffusion coefficients, of the order of $10^{-5} \mathrm{~cm}^{2} \mathrm{~s}^{-1}$ for oilsoluble molecules (like $\mathrm{Br}_{2}$ or the $\mathrm{BrO}_{2}$ radical) and $10^{-6} \mathrm{~cm}^{2}$ $\mathrm{s}^{-1}$ for water-soluble molecules (diffusion coefficient of water droplets) is sufficient to explain the pattern. In this work we show that the presence of large clusters and consequently the presence of a third diffusion coefficient of the order of $10^{-7} \mathrm{~cm}^{2} \mathrm{~s}^{-1}$ are necessary for dashed waves. This finding may require new models that, among other features, can include cross-diffusion between single droplets and clusters.

By significantly extending the lifetime of dashed waves in this system, this procedure may make it possible to construct a photosensitive version of the BZ reaction in which one can study the behavior of dashed waves under different types of forcing.

\section{Acknowledgements}

This work was supported by the MCyT (Spain) under projects FIS2004-03006 and FIS2007-64698, Xunta de Galicia (Spain); under project PGIDIT05PXIC20607PN and the National Science Foundation under grant CHE 0615507.

\section{References}

1 V. K. Vanag and I. R. Epstein, Phys. Rev. Lett., 2001, 87 228301.

2 V. K. Vanag and I. R. Epstein, Phys. Rev. Lett., 2003, 90, 098301.

3 V. K. Vanag and I. R. Epstein, Proc. Natl. Acad. Sci. U. S. A., 2003, 100, 14635-14638.

4 T. H. Wines, A. S. Dukhin and P. Somasundaran, J. Colloid Interface Sci., 1999, 216, 303-308.

5 C. A. T. Laia, W. Brown, M. Almgren and S. M. B. Costa, Langmuir, 2000, 16, 465-470.

6 D. Schubel, O. D. Bedford, G. Ilgenfritz, J. Eastoe and R. K. Heenan, Phys. Chem. Chem. Phys., 1999, 1, 2521-2525.

7 W. Meier, Langmuir, 1996, 12, 1188-1192.

8 R. P. W. J. Struis and H. F. Eicke, J. Phys. Chem., 1991, 95, 5989-5996.

9 N. I. Gerhardt and S. R. Dungan, Biotechnol. Bioeng., 2002, 78, $60-72$.

10 (a) R. Lombardo, C. Sbriziolo, M. L. T. Liveri, K. Pelle, M. Wittmann and Z. Noszticzius, Nonlinear Dynamics in Polymeric Systems, ACS Symp. Ser., 2004, 869, 292-308; (b) M. Pelle, M. Wittmann, Z. Noszticzius, R. Lombardo, C. Sbriziolo and M. L. T. Liveri, J. Phys. Chem. A, 2003, 107, 2039-2047.

11 D. Cuiñas, I. Berenstein, J. Carballido-Landeira and A. P. Muñuzuri, Phys. Rev. E, submitted.

12 (a) V. K. Vanag and D. V. Boulanov, J. Phys. Chem., 1994, 98, 1449-1453; (b) V. K. Vanag and I. Hanazaki, J. Phys. Chem., 1996, 100, 10609-10614; (c) V. K. Vanag and I. Hanazaki, J. Phys. Chem., 1995, 99, 6944-6950. 